MATEC Web of Conferences 48,01001 (2016)

DOI: $10.1051 /$ matecconf $/ 20164801001$

CC Owned by the authors, published by EDP Sciences, 2016

\title{
Design and analysis of power supply and information transfer to three-axis gyroscope stabilizer platform
}

\author{
Lev Belyanin ${ }^{1, a}$, Doan Ket $\mathrm{Vu}{ }^{1}$, Timofey Pozharskiy ${ }^{1}$ and Trong Yen Nguyen ${ }^{2}$ \\ ${ }^{1}$ National Research Tomsk Polytechnic University, 634050 Tomsk, Russia \\ ${ }^{2}$ Science and Technology Institute of Military, No. 17 Hoang Sam, Nghia Do, Cau Giay District, Hanoi, Viet Nam
}

\begin{abstract}
The paper focuses on problems of the engineering implementation of 3-axis gyrostabilizer of the camera for orthophotography performed from light and ultralight aircrafts. The functional diagram of gyrostabilizer is characterized by a lighter ball-bearing having a large inner diameter used for the platform suspension on its internal (vertical) axis. The problem is to transfer the supply voltage to the platform and the electric signals to and from the platform. Design solutions concerning the power supply and information transfer are supported by the experimental techniques suggested in this paper, namely: the use of the rolling contact; three-phase gyro motor power supply replaced by that from the single-phase network; signal transmission from the precession angle sensor using the air transformer; current and voltage frequency division; DC mode selection for the torquer. The results obtained prove the efficiency of the suggested design solutions.
\end{abstract}

\section{Introduction}

A three-axis gyroscopic stabilizer is intended for the digital camera stabilization to produce orthophotography from light and ultralight aircrafts, such as helicopters, airplanes and trikes. A vertical stabilization of the camera is necessary to generate a true vertical photograph taken with its optical axis such that an aircraft's position coordinates detected by the GPS or GLONASS receivers would correspond to the centre of each aerial photograph. An azimuth-stabilized camera, i.e. its vertical stabilization, firstly, improves the quality of aerial photographs and, secondly, creates the conditions for generating a continuous picture of the Earth surface. This gyroscopic stabilizer incorporated on aircraft can be simultaneously used as its course detector. The latter is important especially when a regular course device is not available on board.

Digital cameras are currently installed on gyroscopic stable reference platforms to produce orthophotography from light and ultralight and, hence, inexpensive aircrafts $[1,2]$.

Orthophotography is used in carrying out cadastral works, pipeline, highway and railway examination, and others.

\footnotetext{
${ }^{\text {a }}$ Corresponding author : belyaninln@tpu.ru
} 


\section{Schematic of gyroscopic stabilizer}

The suggested gyrostabilizer is based on the principle of the powered gyroscopic stabilization using three restrained gyros having a ball-bearing suspension. The functional diagram of the gyrostabilizer is given in Figure 1 that does not include the camera.

The gyrostabilizer is characterized by the series stabilization circuit that stabilizes the internal frame of gimbal suspension using the two-gyro leveling system. A uniaxial stabilizer with the normal stabilized axis is placed on the internal frame. The platform suspensions are represented by the lighter ball-bearing having a large inner diameter.

In terms of the problem solution, this schematic is considered to be optimum due to the following reasons:

- to provide stabilization, only one restrained gyro should be placed on the platform. Thus, the residual space allows installing the camera;

- the camera lens is placed in the opening in the centre of the platform and available to an operator;

- coordinate resolver is not required;

- a Y-shaped cantilever used in the capacity of the external frame allows reducing the weight and dimensions of stabilizer and clear a space from parts and units in front of the camera lens.

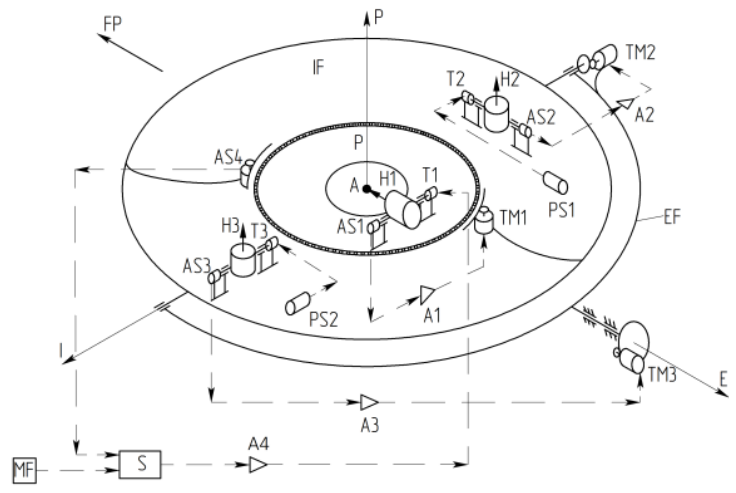

$P$ - platform; $I F$ - internal frame of gimbal suspension; $E F$ - external frame of gimbal suspension; $G$ - gyros; $A S$ - angular direction sensors; $T$ - torquers; - vectors of kinetic moment; $P S$ - pendulum sensors; $A$ - amplifiers; $T M$ - torque motors; $M F$ magnetic field sensor; $S$-summer; $A P, A I$, $A E$ - suspension axes of platform, internal and external frames, respectively; $F P$ flight path.

Figure 1. Functional diagram of gyrostabilizer.

In the stabilization channel of azimuth-stabilized platform, the gyrostabilizer is corrected by the magnetic field sensor placed beyond the gyrostabilizer.

The gyrostabilizer unit includes: gyrostabilizer proper; electronic assembly; magnetic field sensor in azimuth correction system.

The electronic assembly comprises the secondary power supply; power supply converter of gyros, angular direction sensors and torquers; torque motor amplifiers with correction circuits; azimuth correction system of the platform.

\section{Problem definition}

The gyroscopic syabilizer is desiged to operate at roll and pitch angles not exceeding 30 degrees. Therefore, flexible leads placed inside the hollow semi-axes of suspension are used on its axes of the external and internal frames. The angle of the platfrom rotation is not restricted relative to the internal frame, hence the flexible leads cannot be used on the platform suspension axis. In this case, sliding current collectors are conventionally used, which is a combination of slip rings placed on the axis of rotation and current collectors adjusted to them [3]. With a view to reduce the friction torque arising in this sliding current collector and increase its wear resistance, the diameter of slip rings is selected as less as possible (in real gyroscopic instruments, the diameter of slip rings comes to millimeter units in the best models of current collectors). 
Even a shallow analysis of the schematic presented in Figure 1, shows the impossibility of using sliding current collectors on the suspension axis of the platform due to the large diameter of slip rings and their large number. In case of the traditional materials and values of contact pressures used in the construction of the sliding current collectors, the slip ring diameter increased up to $130-150 \mathrm{~mm}$ enables the increase of the friction torque almost by an order. The friction torque in suspensions, sliding current collector, and torque motor gear is the main disturbing factor that reduces the accuracy of the azimuth-stabilized platform. Hence, the success in the whole project depends on the successful design solution of sliding current collectors.

This paper presents results of the development and investigation of the platform power supply, signal transmission from the platform to the gyrostabilizer base and from the internal frame to the platform.

\section{Main design solutions}

According to Figure 1, the restrained gyro is placed on the platform by the suspension axis of which the precession angle sensor and torquers are installed. Let us give the important characteristics of the gyro motor and sensors.

The asynchronous gyro motor operates on a $36 \mathrm{~V}$ alternating current (AC) $400 \mathrm{~Hz}$ three-phase power supply.

The induction precession angle sensor has the exciting winding which operates on a 36VAC 400 Hz power supply.

The AC electromagnetic torquer has the exciting windings which also operate on a $36 \mathrm{VAC} 400 \mathrm{~Hz}$ power supply. AC voltage $400 \mathrm{~Hz}$ supplied to the control winding is phased with the voltage supplied to the exciting winding.

Since the exciting windings are supplied by voltages similar to that of the gyro motor, seven circuits of the current transfer are required to provide the functioning of the platform azimuthstabilizing and correcting channels.

Therefore, the authors suggest the following.

First, to provide only two circuits for the electric coupling between the platform and the internal frame. To implement one of them (case circuit), a ball-bearing of the platform suspension is suggested which can successfully perform this function due to the large number of balls. The second circuit is based on a rolling contact, i.e. an assembly comprising a ring with a large diameter and a roller adjusted to it. This roller is bound to be rotated in its supports with the minimum friction and has a proper electrical connection with its holder. These circuits provide the simultaneous of 36VAC 400 $\mathrm{Hz}$ single-phase and direct current (DC) power supply for the torquer control. It is advisable to use coupling capacitors and inductors to provide the efficient frequency division of AC and DC supply voltage.

Second, the gyro motor three-phase power supply is advisable to replace by an AC single-phase power supply using a phase-shifting capacitor. The experience of this application is described in works $[4,5,6]$.

Third, to provide DC operation for the torquer to allow the exciting winding to operate on a DC rectifier. The latter is placed on the platform. Experiments show that the operation of this DC electromagnetic torquer is much better than that on AC supply.

Fourth, the wireless transmission system should be used to transmit the AC output signal from the angular direction sensor to the base of the torque motor amplifier and then to its input.

The description of the experimental facility designed and manufactured to prove the efficiency of suggestions and their experimental studies is given in Section 5. 


\section{Experiments}

Figure 2 presents the electric schematic diagram of the experimental facility intended for the investigation of the rolling contact with the frequency division of supply voltage and control signals transmitted.

Low resistor $R_{a}$ is series-connected to the control winding of the torquer and intended for the oscillographic measurements of the current in this winding, including the control current variable.

The experimental facility is based on a tilt stand the platform of which arranges the slip ring, restrained gyro with the angular direction sensor and the torquer, and cells arranged in accordance with the electric schematic diagram shown in Figure 2.

The roller is mounted in the arm-holder of the journal bearing. The slip ring, roller, and its holder are made of the type LS-59-1 brass. The diameters of the slip ring contact surface, the roller, and the pivot of the journal bearing are respectively 220,10 , and $2,5 \mathrm{~mm}$. The roller is adjusted to the slip ring by the arm-holder also mounted to the journal bearing and a spring on the support. The spring force is screw-adjusted.

Allowing for the active power generation, transmission coil TC was replaced by its equivalent - a fixed resistor.

Experimental research is aimed at the detection of:

- the contact resistance of the sliding current collector at different rotation rates of the platform and different forces of adjusting the roller to the slip ring;

- the quality of the frequency division of AC and DC supply voltage of the torquer and optimization of the network parameters;

- currents and voltages at the representative points of the diagram.

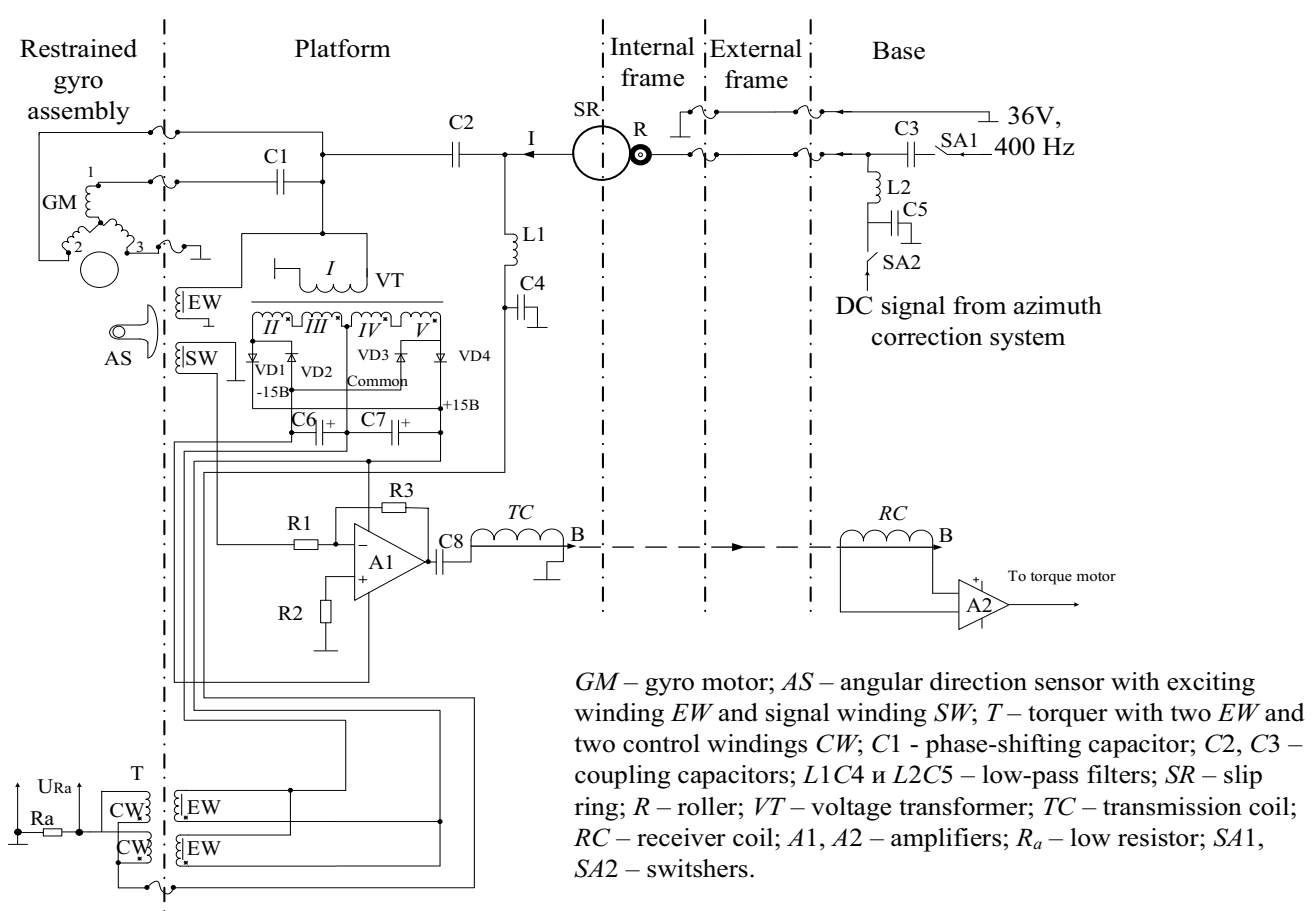

Figure 2. Electric schematic diagram of the experimental facility intended for the rolling contact investigation.

To detect the contact resistance $R_{c}$, the direct current is passed through the sliding current collector, while the loss of voltage $U_{c}$ is registered by an oscillograph (Fig. 3). In case the current $I$ is constant, the input voltage $U$ will be proportional to the contact resistance $R_{c}$. 


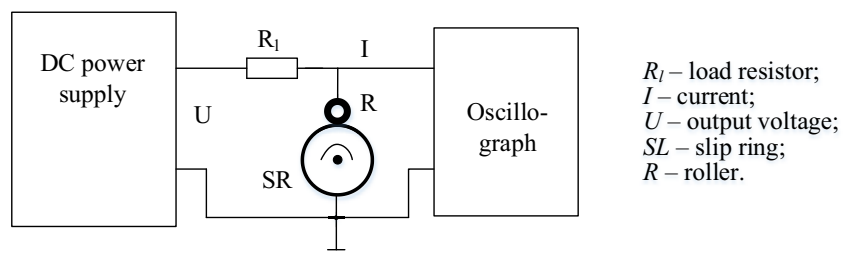

Figure 3. Schematic of contact resistance.

In order to provide the direct current passing through the sliding current collector, i.e. its supply from the current generator, the high output voltage $U$ is used. The load resistor $R_{l}$ is seriesly connected to the circuit. Voltage $U_{c}$ is then obtained from

$$
U_{c}=\frac{U}{R_{l}+R_{c}} \cdot R_{c}
$$

Since $R_{l}>>R_{c}$ then

$$
R_{\mathrm{c}} \approx \frac{R_{1}}{U} \cdot U_{\mathrm{c}}
$$

The type GUA-2D gyro unit is used in the experimental facility. The following experimental parameters are used: $1 \mu \mathrm{F}$ phase-shifting capacitance $C 1 ; 50 \mu \mathrm{F}$ coupling capacitance $C 2 ; 44 \mu \mathrm{F}$ coupling capacitance $C 3 ; L 1=L 2=5 \mathrm{H}$ inductance of filter inductor; $C 4=C 5=9,4 \mu \mathrm{F}$ filter capacitance.

At voltage $U=62 \mathrm{~V}$ (Fig. 3) supplied through the load resistor of $R_{1}=280 \mathrm{ohm}, I=0,22 \mathrm{~A}$. The contact resistance is detected at different values of the adjustmnet force and different rates of the platform rotation. Figure 4 presents the oscillogram of the contact resistance that reflects its behavior in time during the platform rotation. For comparison, Figure 4 contains the curve of the static contact resistance $R_{s t}$.

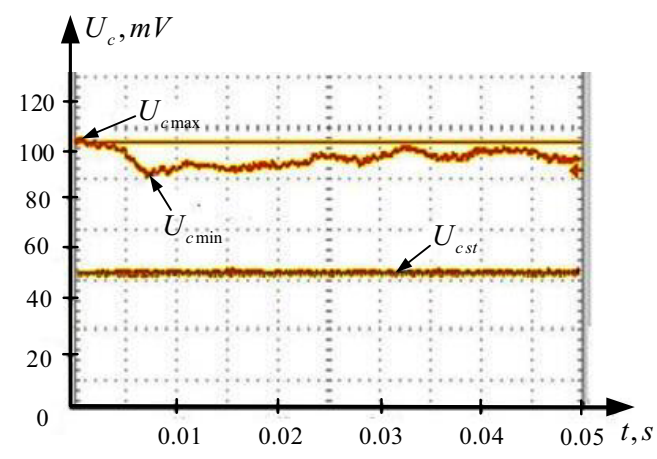

Figure 4. Contact resistance curves.

Research findings are given in Table 1 that includes the following notations: $F$-adjustment force; $R_{s t}$ - static contact resistance; $\omega$ - angular rate of rotation of the slip ring; $R_{\min }$ and $R_{\max }-$ minimum and maximum contact resistances within the range of which the slip ring is changed at its rotation.

According to Table 1, the slip ring rotation increases the contact resistance by $1.7 \div 2.6$ times depending on the values of the adjustment force and the angular rate of rotation. The quality of contact is enhanced with the increase of the adjustment force and the decrease of the angular rate of rotation. 
During the ring rotation, the contact resistance ranges between $0.05 \div 0.1 \mathrm{ohm}$ that is quite acceptable in terms of the given problem solution.

Table 1. Experimental findings for contact resistance.

\begin{tabular}{|c|c|c|c|c|c|}
\hline$F, \mathrm{G}_{\mathrm{s}}$ & $R_{c}, \mathrm{ohm}$ & $\omega, \mathrm{dps}$ & $R_{\text {min }}, \mathrm{ohm}$ & $R_{\max }, \mathrm{ohm}$ & $\Delta R_{c}, \mathrm{ohm}$ \\
\hline \multirow{3}{*}{100} & \multirow{3}{*}{0.385} & 20 & 0.873 & 0.946 & 0.073 \\
\hline & & 60 & 0.914 & 0.986 & 0.073 \\
\hline & & 120 & 0.914 & 0.986 & 0.073 \\
\hline \multirow{3}{*}{150} & \multirow{3}{*}{0.287} & 20 & 0.568 & 0.623 & 0.055 \\
\hline & & 60 & 0.586 & 0.677 & 0.091 \\
\hline & & 120 & 0.718 & 0.827 & 0.109 \\
\hline \multirow{3}{*}{200} & \multirow{3}{*}{0.280} & 20 & 0.464 & 0.536 & 0.073 \\
\hline & & 60 & 0.464 & 0.555 & 0.091 \\
\hline & & 120 & 0.518 & 0.609 & 0.091 \\
\hline \multirow{3}{*}{250} & \multirow{3}{*}{0.250} & 20 & 0.446 & 0.518 & 0.073 \\
\hline & & 60 & 0.436 & 0.546 & 0.109 \\
\hline & & 120 & 0.505 & 0.596 & 0.091 \\
\hline \multirow{3}{*}{300} & \multirow{3}{*}{0.254} & 20 & 0.427 & 0.482 & 0.055 \\
\hline & & 60 & 0.500 & 0.555 & 0.055 \\
\hline & & 120 & 0.500 & 0.573 & 0.073 \\
\hline \multirow{3}{*}{350} & \multirow{3}{*}{0.236} & 20 & 0.418 & 0.473 & 0.055 \\
\hline & & 60 & 0.455 & 0.509 & 0.055 \\
\hline & & 120 & 0.486 & 0.541 & 0.054 \\
\hline
\end{tabular}

Investigations of the power supply system of the gyro motor and other devices located on the platform shown in Figure 3, show the efficiency of design solutions implemented in the experimental facility.

Thus, the starting time of the gyro motor comes to $2 \mathrm{~min}$ and $55 \mathrm{~s}$. The total consumption of the current passing through the sliding current collector is 0.22 A. Voltage losses on the coupling capacitors $C 2$ and $C 3$ are 1 and $1.3 \mathrm{~V}$ respectively. The current variable, i.e. an interference in the control winding of the torquer, is $0.247 \mathrm{~mA}$ at $10 \mathrm{~mA}$, the maximum design value of the current correction.

\section{Design and investigation of wireless transmission system}

According to the functional diagram of gyrostabilizer, the signal transmission from the precession angle sensor placed on the platform is bound to be arrived to the input of the amplifier of the torque 
motor incorporated in the electronic assembly. The signal is transmitted from the platform to the base by means of the wireless transmission system, and further to the electronic assembly across the wire.

The following wireless transmission techniques are known: radio channel, optical channel, acoustic channel, variable magnetic field. The selection of the in-depth analysis technique and investigations was conditioned by the fact that the transmitted signal is an amplitude-modulated AC signal at $400 \mathrm{~Hz}$ carrier frequency.

The main requirements for the wireless transmission are as follows:

- stability of the transmission factor;

- no signal distortion;

- noiseimmunity;

- easiness of implementation in particular conditions.

The latter teqchnique was selected for further investigations. It is based on the use of the mutual induction of the transmission and receiver coils situated next to each other [7, 8]. The transmission coil placed on the gyrostabilized platform, accepts the enhanced signal from the precession angle sensor. The receiver coil placed on the gyrostabilizer base, generates the electromotive force that can be obtained from [9]:

$$
e_{2}=-\frac{d}{d t}\left(L_{2} \cdot i_{2} \pm M \cdot i_{1}\right)
$$

where $e_{2}$ is the electromotive force in the receiver coil; $L_{2}$ is the induction in the receiver coil; $M$ is the mutual induction of coils; $i_{1}$ and $i_{2}$ are currents passing through coils.

In (3), the second component is the electromotive force of transformation, while the second is the electromotive force of self-induction. In case of a rather high input resistance of the amlifier, the first component is neglected.

Thus, the properties of the wireless transmission system mostly depend on the value and stability of the mutual induction of coils.

The induction depends on the geometry and the number of turns in both transmission and receiver coils, their mutual arrangement, and the environmetal conditions under which the variable magnetic field propagates, i.e. magnetic permeability and electrical conductivity.

The stability of the transmission factor in the wireless transmission system determines the stability of the transmission factor in the open circuit of stabilization loop in azimuth that ensures the accuracy of the system stabilization and consistency.

Theoretical studies and quantification of the different factors affecting the value of the mutual induction turned to be inefficient. Therefore, much experimetal research has been carried out into the following issues:

- to arrange the transmission and receiver coils in the gyrostabilizer such that to provide the minimum dependence between the mutual induction and the spatial position of the platform and gyrostabilizer base;

- to study the effect on the system operation exerted by metal parts and assemblies of gyrostabilizer that are situated nearby the transmission and receiver coils;

- to determine the value of the output resistance of the system as a source of signal.

To solve the first problem, a transmission coil and two variants of receiver coils were designed and manufactured.

The transmission coil was designed allowing for its mount position, i.e. on the platform under the ball-bearing suspension, close to it. The physical configuration of this coil is given in Figure 5 .

The first variant of the receiver coil is a combination of four coils with series and cumulative connection placed on the base on the edge of the opening that gives the camera a field of view. The idea is to properly arrange the coils, thereby lowering the dependence between the total electromotive force of coils and the rotation of the transmission coil relative to the base. It is supposed that while the mutual induction of a receiver and a transmision coil increases at rotation, the induction of another 
coil placed on the other side of the opening decreases and vice versa. At the same time, this experiment shows the optimum coil arrangement relative to the base and the radius of the opening.

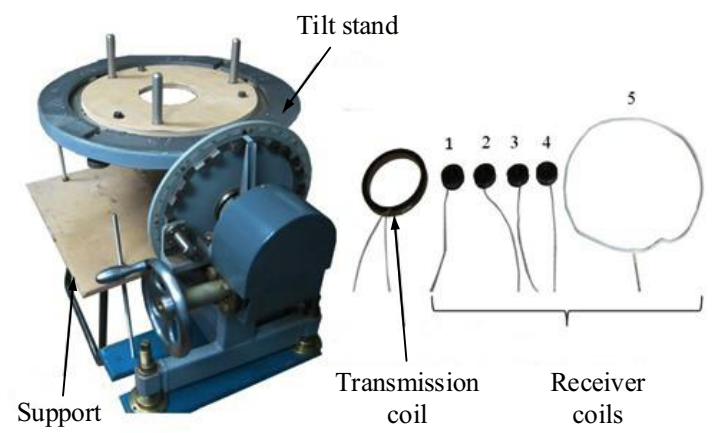

Figure 5. General view of the tilt stand and a set of coils.

The second variant of the receiver coil implies its increased diameter such that the total magnetic flux created by the transmission coil and tying the turns of the receiver coil, would less depend on the relative angular oscillations of coils. In Figure 5, it is the self-supported coil 5. The coil parameters are given in Table 2.

Table 2. Parameters of coils.

\begin{tabular}{|c|c|c|c|c|c|}
\hline Coil types & $\begin{array}{c}\text { Winding wire } \\
\text { diameter, } \mathrm{mm}\end{array}$ & $\begin{array}{c}\text { Number } \\
\text { of turns }\end{array}$ & $\begin{array}{c}\text { Inner diameter } \\
\text { of coil, } \mathrm{mm}\end{array}$ & Resistance, ohm & Induction, $\mathrm{mH}$ \\
\hline Transmission coil & 0,4 & 172 & 109,3 & 9,1 & 6,82 \\
\hline Coil 1 & 0,1 & 650 & 30,3 & 155 & 19,1 \\
\hline Coil 2 & 0,1 & 650 & 30,3 & 156 & 19,1 \\
\hline Coil 3 & 0,1 & 650 & 30,3 & 159 & 19,7 \\
\hline Coil 4 & 0,1 & 650 & 30,3 & 155 & 19,1 \\
\hline Coil 5 & 0,14 & 150 & 310 & 216 & 34,1 \\
\hline
\end{tabular}

Experiments on the optimization of the wireless transmission system are carried out using the uniaxial tilt stand shown in Figure 5. The transmission coil is mounted to the disk made of nonmagnetic and non-conductive material. The disk, in turn, is mounted to the platform of the tilt stand. Receiver coils arranged on the support are also made of non-magnetic and non-conductive material. The distance between the transmission coil and the support is $240 \mathrm{~mm}$ that is equal to the distance between the coil and the gyrostabilizer base. Harmonic voltage $400 \mathrm{~Hz}$ is supplied to the transmission coil. The voltage of $3 \mathrm{~V}$ is selected to provide $1 \mathrm{~W}$ power generated by the coil. This voltage is constant. Receiver coils transmit voltage to the oscillograph and the AC electronic millivoltmeter. In each experiment, the transmission factor $F$ is obtained as the ratio between the real value of voltage $U_{2}$ of receiver coils and that of the transmission coil:

$$
F=\frac{U_{2}}{U_{1}}
$$

The transmission factor variation is presented in Figure 6 and has the following conditions. Four coils 1-4 shown in Figure 5, have a series and comulitive connection. The coil planes have different angles $\alpha$ of inclination relative to the support. The coils are mounted to the support in the centres of 
the four sides of the square. The square side equals to the diameter of the opening in the centre of gyrostabilizer. At a change in the angle of inclination of coils, their axes stay in the plane normal to the support that contains the opening axis.

The maximum value of transmission factor and its maximum independence of the angle of inclination $\beta$ of the transmission coil are acheved at $\alpha=90^{\circ}$, i.e. at the normal position of planes of coils 1-4 relative to the support. Then, the maximum change in the transmission factor is $4,2 \%$ at $\beta$ angle of inclination ranging within $\pm 30^{\circ}$.

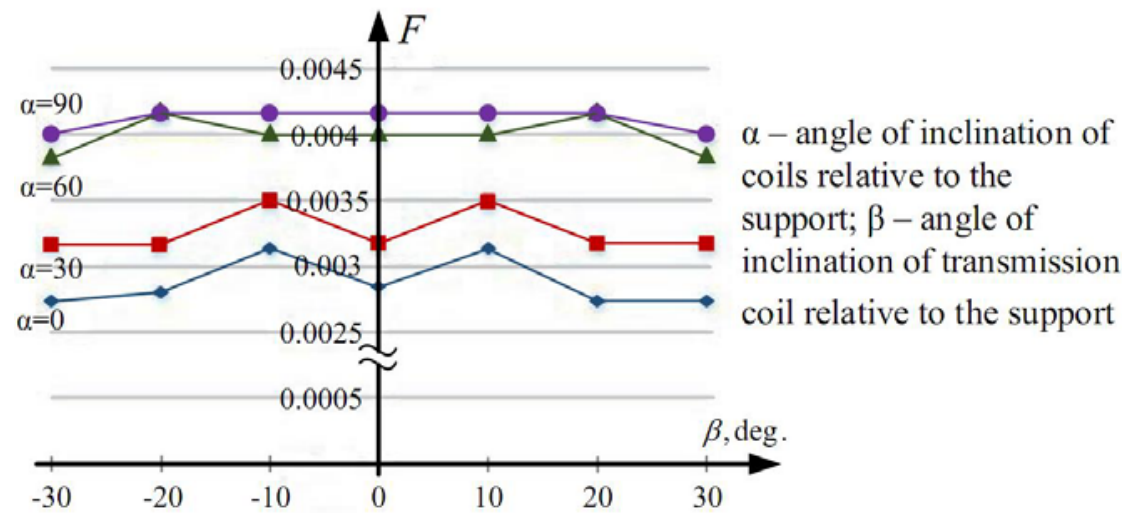

Figure 6. Transmission factor curves.

Experiments conducted for the self-supported coil 5 coaxially mounted to the support opening show the great stability of the transmission factor as well as its greater value. Its maximum changes come to $3,4 \%$ at $\beta$ angle of inclination within the same range.

Since the second variant of the system with the self-supported coil provides the stability of transmission factor and is easier in its implementation, it is used in further experiments.

The dependence between the receiver coil voltage and the load current is obtained allowing to detect the internal resistance of the wireless transmission system as $222 \mathrm{ohm}$. In this experiment, the power supply has a low internal resistance. In case the transmission coil is supplied by the amplifier of the angular direction sensor, the internal resistance rather increases.

The experimental studies of the wireless transmission system are accomplished by the estimation of its behavior affected by metal parts and assemblies arranged nearby the transmission and receiver coils and between them.

As is known [10], the interaction between the variable magnetic field and metal is conditioned by the field frequency and metal properties, such as magnetic permeability and electrical conductivity. Magnetic permeability modifies the nature of the field/metal interaction at zero and low frequency. Magnetic field permeates through metal and becomes stronger in it. In this case metal acts as a magnetic circuit. At medium and high frequencies the nature of this interaction changes, and electrical conductivity becomes increasingly important. The variable magnetic field enables the electromotive force in metal mass resulting in eddy currents. The latter create their own variable magnetic field that interacts with the external field, therby weakening it in metal mass and strengthening beyond it.

In other words, the variable magnetic field created by the transmission coil is affected by any metal parts and assemblies that can lead to the change of transmission factor of the wireless system or unwanted phase shift between the voltage supplied to the transmission coil and that released by the receiver coil.

A ball-bearing with a large diameter is placed on the transmission coil side opposite to the receiver coil, that is used for the platform suspension. The transmission factor then decreases by $12 \%$.

Two steel sheets of $\approx 0.5 \mathrm{~kg}$ mass each are placed on the support nearby the reciever coil. The transmission factor then decreases by almost the same percentage. 
The steel sheet $2 \mathrm{~mm}$ thick and area exceeding that covered by the receiver coil is placed between the coils. The wireless transmission system operates properly, however, the transmission factor decreases then by $37 \%$.

As for non-magnetic metals such as aluminum and copper alloys placed nearby the coils, they have a small effect on the wireless transmission system.

These experimental findings prove the efficiency of the suggested wireless transmission system for the signal generated by the angular direction sensor. Probably, this is owing to the frequency value of signaling, i.e. $400 \mathrm{~Hz}$. This frequency allows the air transformer formed by the two coils to consistently operate. However, the interaction between the variable magnetic field and conductive materials is still insufficient.

\section{Conclusion}

Research results proved the efficiency of the suggested design solutions that allows giving recommendations on their application to the gyrostabilizer and in other similar designs.

Experiments on the determination of the friction torque generated by the rolling contact have not been conducted. Taking into account the fact that the friction torque was mainly produced by the journal bearings of the roller suspension, the roller was mounted to its holder using ball-bearings, while the electric coupling between them was achieved by central point contacts. At a time, with a view to reduce the contact resistance and increase the contact reliability, two similar rollers were installed completely opposite to the axis of the platform suspension.

\section{References}

1. L. Cao, L. Hongbo, G. Kemper, ISPRS Annals 37, 1667 (2008)

2. G. Kemper, ISPRS Annals 39, 351 (2012)

3. E. Nikitin, S. Shestov, V. Matveev, Gyro systems: gyroscope elements (Vysshaya Shkola Publ., Moscow, 1988)

4. Y. Nguyen, K. Vu, Proc. $18^{\text {th }}$ Int. Sci. Conf. 'Modern Engineering and Technology 1, 381 (2012)

5. L. Belyanin, L. Gurin, V. Martemyanov, S. Samoylov, RF Patent 2003045 (1993)

6. L. Belyanin, A. Golikov, V. Martemyanov, S. Samoylov, Coll. Papers OAO 'Geofit' 2, 34 (2002)

7. S. Cheng, P. David, Sel. Papers Int. $10^{\text {th }}$ Worksh. Micro and Nanotech. Power Gen. and Energy Convers. Appl. 61 (2010)

8. J. Hirai, T. Kim, A. Kawamura, IEEE Trans. on Indust. Electronics 46, 349 (1999)

9. L. Bessonov, Teoreticheskie osnovy elektrotekhniki. Elektricheskie tsepi [Electrical Engineering Theory. Electric circuits] (Vysshaya Shkola Publ., Moscow, 1996)

10. A. Nenashev, Konstruirovanie radioelektronnykh sredstv [Radioelectronic equipment design] (Vysshaya Shkola Publ., Moscow, 1996) 